\title{
Multicolor Stationary Light
}

\author{
Yi Chen ${ }^{1}$, Serguei Andreevich Moiseev ${ }^{2}$ and Byoung Seung Ham ${ }^{1}$ \\ ${ }^{1}$ Center for Photon Information Processing, and the Graduate School of IT, Inha University \\ ${ }^{2}$ Kazan Physical-Technical Institute of Russian Academy of Sciences \\ ${ }^{1}$ South Korea \\ ${ }^{2}$ Russia
}

\section{Introduction}

Quantum coherence and interference (Scully \& Zubairy, 1997) are leading edge topics in quantum optics and laser physics, and have led to many important novel effects, such as coherent population trapping (CPT) (Arimondo \& Orriols, 1976; Alzetta et al., 1976; Gray et al., 1978), lasing without inversion (LWI) (Harris, 1989; Scully et al., 1989; Padmabandu et al., 1996), electromagnetically induced transparency (EIT) (Boller et al., 1991; Harris, 1997; Ham et al., 1997; Phillips et al., 2003; Fleischhauer et al., 2005; Marangos, 1998), high refractive index without absorption (Scully, 1991; Scully \& Zhu 1992; Harris et al., 1990), giant Kerr nonlinear effect (Schmidt \& Imamoglu, 1996), and so on. In particular, EIT, which can dramatically modify the absorption and dispersion characteristics of an optical medium, plays an important role in quantum optics. In the last two decades, EIT has attracted great attention and has been successfully applied to ultraslow and stopping light (Kasapi et al., 1995; Hau et al., 1999; Kocharovskaya et al., 2001; Liu et al., 2001; Turukhin et al., 2002), quantum switching (Ham \& Hemmer, 2000), quantum memory (Ham et al., 1997; ibid., 1998), quantum entanglement generation (Lukin \& Imamoglu, 2000), and quantum computing (Lukin \& Imamoglu, 2001). It is well established that light is the fastest information carrier in nature. However, controlling light for localized application is very difficult. Thus, manipulation of light velocity becomes a crucial task in optical and quantum information processing (Nielsen \& Chuang, 2000). Recently light localization using EIT has been demonstrated for stationary light (Bajcsy et al., 2003). Stationary light gives novel effects to nonlinear quantum optics in the context of lengthening light-matter interaction time. Compared with ultraslow light, where the medium's length is a limiting factor, stationary light is free from spatial constraint. For example, the interaction time using ultraslow light in a semiconductor quantum dot, whose spatial dimension is less than a few tens of nanometers, is much less than nanosecond. By using a stationary light technique, however, we can enormously increase the interaction time of the light with such a nano optical medium. In this chapter, we discuss stationary light based on the EIT-induced ultraslow light phenomenon. We theoretically investigate how to dynamically manipulate multicolor (MC) stationary light in the multi double lambda-type system by simply changing the parameters of control fields, and demonstrate ultralong trapping of light, which is different from the conventional quantum mapping phenomenon. Quantum coherent control of the stationary light has potential applications to various quantum optical processing such as quantum nondemolition measurement and quantum wavelength conversion.

Source: Advances in Lasers and Electro Optics, Book edited by: Nelson Costa and Adolfo Cartaxo, ISBN 978-953-307-088-9, pp. 838, April 2010, INTECH, Croatia, downloaded from SCIYO.COM 
This chapter is organized as follows. In section 2, quantum coherent control of two-color stationary light is described. In section 3, quantum manipulation of MC stationary light is presented. In section 4, we give the results of MC stationary light, with discussions. Finally, section 5 offers conclusions.

\section{Quantum coherent control of light}

\subsection{Model and theory}

In this section, we present dynamic control of two-color stationary light in a double-lambda type four-level system using EIT. Fig. 1 shows the energy level diagram. We assume that initially one weak probe quantum field $E_{+}$which couples the transition $|1\rangle-|3\rangle$ enters the medium in a forward propagation direction $\mathrm{k}_{+}$, where all the atoms are in the ground state $\mid 1>$. Under the influence of classical control field $\Omega_{+}$, which is resonant with the transition $|2\rangle-|3\rangle$ and propagates in the same direction $\kappa_{+}$, the probe field propagates at an ultraslow group velocity. After the probe pulse enters the medium completely, we adiabatically switch on the second control field $\Omega_{-}$, which is resonant with the transition |2> - $\mid 4>$ and propagates in the backward direction $\kappa$. The probe pulse will be almost stationary if the stationary light condition is satisfied. The propagation direction $\mathrm{k}_{-}$of the field $E_{\text {- }}$ is determined by phase matching with the Bragg condition: $\mathrm{k}_{-}=\mathrm{k}_{+}-\kappa_{+}+\kappa_{-}$. Here $\Omega_{+}$and $\Omega_{-}$are the Rabi frequencies of two control fields. Below we will study the evolution of the stationary light when (1) $\Omega_{-}$is switched off after a certain time while keeping the control filed $\Omega_{+}$on, and (2) $\Omega_{+}$is switched off after a certain time while keeping the control filed $\Omega_{-}$on (see Fig. 2).
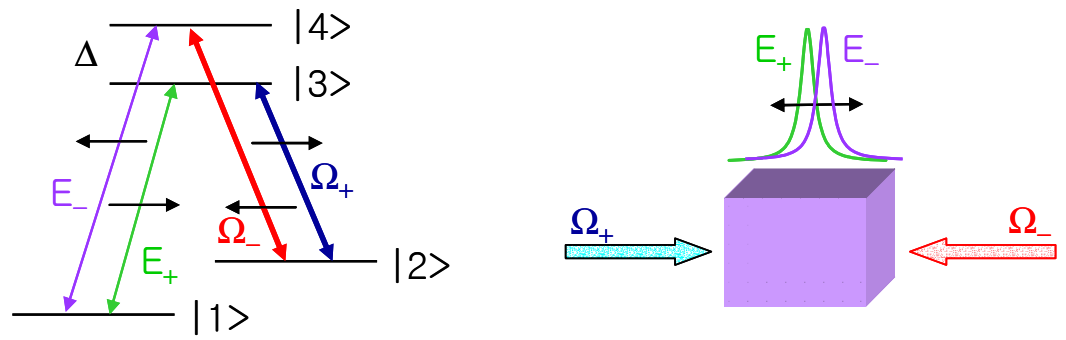

Fig. 1. Energy level diagram for two-color stationary light.

For theoretical analytical purposes, we introduce a quantum field $E_{\sigma}=\sqrt{\hbar \omega_{\sigma} /\left(2 \varepsilon_{0} V\right)} A_{\sigma} e^{-i \omega_{\sigma}(t-\sigma z / c)}+H . C$., where $\sigma=+/-$ stands for forward/backward field, respectively, which is traveling in a $\pm z$ direction. $A_{\sigma}$ is the slowly varying field operator, $\hbar$ is the Planck's constant, and $\varepsilon_{0}$ is the electric permittivity. We assume that the quantization volume $\mathrm{V}$ is 1 . Under the rotating-wave approximation, we obtain the following Hamiltonian for the quantum fields and atoms in the interaction picture:

$$
\begin{aligned}
& H=\hbar g_{+} \sum_{j=1} A_{+}\left(t, z_{j}\right) P_{31}^{j} e^{i \omega_{+} z_{j} / c}+\hbar g_{-} \sum_{j=1} A_{-}\left(t, z_{j}\right) P_{41}^{j} \mathrm{e}^{-\mathrm{i} \omega_{-} z_{j} / c} \\
& -\hbar \sum_{j=1}\left\{\Omega_{+} P_{32}^{j} \exp \left[i\left(K_{+} z_{j}+\varphi_{+}\right)\right]+\Omega_{-} P_{42}^{j} \exp \left[-i\left(K_{-} z_{j}-\varphi_{-}\right)\right]\right\}+H . C_{.},
\end{aligned}
$$


where $P_{n m}^{j}=\left(P_{m n}^{j}\right)^{+}$are the atomic operators, $g_{\sigma}=\wp_{\sigma} \sqrt{\omega_{\sigma} /\left(2 \varepsilon_{0} \hbar V\right)}$ is the coupling constant of photons with atoms, $\wp$ is the dipole moment for each transition, and $\varphi_{\sigma= \pm}$ are the phases of the control fields, $\omega_{+}=\omega_{31}$ and $\omega_{-}=\omega_{41}$. Using Hamiltonian (1) and adding the decay constants $\gamma_{3}, \gamma_{4}$, and $\gamma_{2}$ for the atomic transitions $\left.|1>-| 3\right\rangle,|1\rangle-\mid 4>$ and $|1\rangle-$ |2>, we derive equations for the field $A_{\sigma}$ and atomic operators $P_{m n}^{j}$.

Under the typical adiabatic condition for the slow light propagations (Fleischhauer \& Lukin, 2000; Zibrov et al., 2002), we introduce new field operators $\Psi=e^{-i\left(\phi_{\sigma}+\sigma \omega_{21} z / c\right)} \sqrt{N} g_{\sigma} A_{\sigma} / \Omega_{\sigma}\left(\mathrm{N}\right.$ is atomic density). Due to a weak field $E_{\sigma= \pm}$ under the slow-light propagation $v_{g} \cong c \Omega_{+}^{2} / N g_{+}^{2}<<c$, we ignore the atomic population on the excited levels $\mid 3>$ and $\mid 4>$ and assume slowly varying amplitudes of the laser fields. Then we can obtain the following coupled wave equations for the new field operators:

$$
\begin{gathered}
\left(\frac{\partial}{\partial z}-i \frac{\omega_{21}}{c}\right) \Psi_{+}(\tau, z)=-\xi_{+} \alpha_{-}\left[\Psi_{+}(\tau, z)-\Psi_{-}(\tau, z)\right] \\
-\frac{\partial}{c \partial \tau}\left\{\alpha_{+} \Psi_{+}(\tau, z)+\alpha_{-} \Psi_{-}(\tau, z)\right\}-\left(\gamma_{2}^{\prime} / c\right) \Psi_{+}(\tau, z) \\
\left(\frac{\partial}{\partial z}+i \frac{\omega_{21}}{c}\right) \Psi_{-}(\tau, z)=-\xi_{-} \alpha_{+}\left[\Psi_{+}(\tau, z)-\Psi_{-}(\tau, z)\right] \\
+\left(g_{-} / g_{+}\right)^{2} \frac{\partial}{c \partial \tau}\left\{\alpha_{+} \Psi_{+}(\tau, z)+\alpha_{-} \Psi_{-}(\tau, z)\right\}+\left(g_{-} / g_{+}\right)^{2}\left(\gamma_{2}^{\prime} / c\right) \Psi_{-}(\tau, z)
\end{gathered}
$$

where $\xi_{\sigma}=N g_{\sigma}^{2} / c \gamma$ are the absorption coefficients, $\alpha_{\sigma}=\Omega_{\sigma}^{2} /\left[\gamma_{2}+\Omega_{\Sigma}^{2}\right], \Omega_{\Sigma}^{2}=\Omega_{+}^{2}+\Omega_{-}^{2}$, $\tau=\int_{-\infty}^{t} d t^{\prime}\left[\gamma_{2}+\Omega_{\Sigma}^{2}\right] /\left(g_{+}^{2} N\right)$ is a new time scale, and $\gamma_{2}^{\prime}=N g_{+}^{2}\left[\gamma_{2}+\Omega_{\Sigma}^{2}\right]^{-1} \gamma_{2}$.



(a)

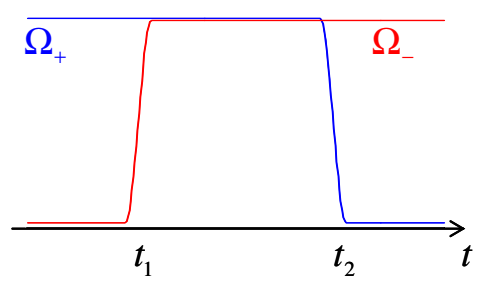

(b)

Fig. 2. Temporal scheme of the switching sequence of the two control fields. (a) $\Omega_{-}$is turned off at $\mathrm{t}=\mathrm{t}_{2},(\mathrm{~b}) \Omega_{+}$is turned off at $\mathrm{t}=\mathrm{t}_{2}$.

Using spatial Fourier transformation $\Psi_{\sigma}(\tau, z)=\int_{-\infty}^{\infty} d k e^{i k z} \tilde{\Psi}_{\sigma}(\tau, k)$, we can find the following analytical solutions of Eqs. (2) and (3):

$$
\begin{gathered}
\Psi_{\sigma}(\tau, z)=\int_{-\infty}^{\infty} d k \exp \left\{+i k z+i \int_{\tau_{o}}^{\tau} d \tau^{\prime} \omega\left(\tau^{\prime}, k\right)\right\} \tilde{\Psi}_{\sigma}\left(\tau_{o}, k\right) \\
\omega\left(\tau^{\prime}, k\right)=i \eta \gamma_{2}^{\prime}-\frac{\operatorname{ck}\left\{\eta\left(\xi_{-} \alpha_{+}-\xi_{+} \alpha_{-}\right)-i k\right\}-i\left(\partial / \partial \tau^{\prime}\right)\left(\eta^{-1} \xi_{-}-i k \tilde{\alpha}\right)}{\left\{\eta^{-1} \xi_{-}-i k \tilde{\alpha}\right\}}
\end{gathered}
$$


where $\tilde{\Psi}_{-}\left(\tau_{o}, k\right)=\tilde{f}_{-+}(k) \tilde{\Psi}_{+}\left(\tau_{o}, k\right), \quad \tilde{f}_{-+}(k)=\left(1+i k / \xi_{+}\right) /\left(1-i k / \xi_{-}\right), \quad \eta=\left(\Omega_{\Sigma}^{2}+\gamma \gamma_{2}\right) \Omega_{\Sigma}^{-2}$ and $\tilde{\alpha}=\alpha_{+}-\left(g_{-} / g_{+}\right)^{2} \alpha_{-}$. The functions $\tilde{\Psi}_{+,-}\left(\tau_{0}, z\right)$ are determined by the initial conditions for the field $A_{+,-}$which enters the medium $\left(\Omega_{-}\left(t<t_{1}\right)=0\right)$. From Eq. (4) we obtain the coupled fields $\Psi_{+}\left(t, z^{\prime}\right)$ and $\Psi_{-}(t, z)$ expressed by nonlocal spatial relations:

$$
\begin{aligned}
& \Psi_{+}(t, z)=\int_{-\infty}^{z+\varepsilon} d z^{\prime} f_{+}\left(z-z^{\prime}\right) \Psi_{-}\left(t, z^{\prime}\right) \\
& \Psi_{-}(t, z)=\int_{z-\varepsilon}^{\infty} d z^{\prime} f_{-}\left(z-z^{\prime}\right) \Psi_{+}\left(t, z^{\prime}\right)
\end{aligned}
$$

where $f_{\sigma}\left(z-z^{\prime}\right)=\xi_{\sigma}\left(1+\frac{\xi_{\sigma}}{\xi_{\sigma^{\prime}}}\right) \eta\left(z^{\prime}-z\right) e^{-\xi_{\sigma}\left(z^{\prime}-z\right)}-\frac{\xi_{\sigma}}{\xi_{\sigma^{\prime}}} \delta\left(z-z^{\prime}\right)\left(\sigma= \pm 1, \sigma^{\prime}=\mp 1, \eta(x \geq 0)=1, \eta(x<0)=0\right.$ and $\varepsilon$ is a small value $\varepsilon \rightarrow 0)$. Eq. (6) points out that spatial quantum correlations between the fields $\Psi_{+}(t, z)$ and $\Psi_{-}\left(t, z^{\prime}\right)$ spread out within the spatial size $\xi_{\sigma}^{-1}$. This means that the fields $\Psi_{+}\left(t, z^{\prime}\right)$ and $\Psi_{-}(t, z)$ can be quantum copies of each other if the spatial size $l$ of $\Psi_{ \pm}\left(t, z^{\prime}\right)$ is larger than $\xi_{\sigma}^{-1}$. Therefore the spatial correlations of quantum fields $\mathrm{E}_{+}$and $\mathrm{E}_{-}$ can be determined by Eq. (6).

Now we study temporal dynamics of the two-color coupled fields. Initially the control field $\Omega_{+} \quad\left(\Omega_{-}=0\right)$ is turn on and a probe pulse $A_{+}$with a Gaussian shape $\left(A_{+}(t, z=0)=A_{+, o} \exp \left\{-t^{2} /\left(2 T^{2}\right)\right\}, A_{+, o}\right.$ and $T$ are the amplitude and temporal duration of the field) enters the medium. The solution of Eq. (4) describes the propagation of the field $\Psi_{+}$, that is, $A_{+}=\left(g_{+}^{2} N\right)^{-1 / 2} \Omega_{+} \Psi_{+} e^{i\left(\phi_{+}+\omega_{21} z / c\right)}$ with an ultraslow group velocity $v_{g} \cong c \Omega_{+}^{2}(0) / N g_{+}^{2}<<c$, and the initial spatial size $l_{o}=v_{g} T$ with an amplitude decay in accordance with $\exp \left\{-\eta \gamma_{2} t\right\}$. Thus, due to the EIT effect, the field $A_{+}$is transparent to the optically dense medium $\left(\xi_{+,-} l_{o}>1\right)$. At time $t=t_{1}$, we turn on the backward control field $\Omega_{-}$. Using the dispersion relation $\omega(k)$ in Eq. (5), we obtain the group velocity for the interacting fields $A_{+}$and $A_{-}$:

$$
v=-\left[\left(\Omega_{\Sigma}^{2}+\gamma_{2}\right) / N g_{+}^{2}\right] \partial \omega /\left.\partial \mathrm{k}\right|_{k=0}=c \eta^{2}\left[g_{-}^{2} \Omega_{+}^{2}-g_{+}^{2} \Omega_{-}^{2}\right] /\left[N\left(g_{+} g_{-}\right)^{2}\right]
$$

From Eq. (7), the stationary light condition $(v=0)$ can be derived:

$$
\Omega_{+} / g_{+}=\Omega_{-} / g_{-}
$$

Thus the group velocity of the coupled light can be easily controlled by manipulating the control field Rabi frequencies. If the stationary light condition is satisfied, the two coupled lights can be completely stopped. Ignoring small terms proportional to $c k^{3} / \xi_{-}^{2}$ in Eq. (5), we find the approximate solution of the integral (Eq. (4)) for arbitrarily varying amplitudes of the control laser fields:

$$
\begin{aligned}
A_{\sigma}(\tau, z) & =\frac{l_{o}}{c B(\tau)} \frac{\eta(\tau)\left(\Omega_{\sigma}(\tau) g_{+}\right)}{\eta(0)\left(\Omega_{+}(0) g_{\sigma}\right)} A_{+, o} \\
& \times \exp \left\{-\int_{0}^{\tau}\left(\eta \gamma_{2}^{\prime}\right) d \tau^{\prime}-\left(\beta_{\sigma}(\tau)-z / c\right)^{2} /\left(2 B^{2}(\tau)\right)\right\} \exp \left\{i\left(\phi_{\sigma}+\sigma \omega_{21} z / c\right\}\right.
\end{aligned}
$$


where

$$
\begin{gathered}
\beta_{+}(\tau)=\int_{0}^{\tau} d \tau^{\prime} M_{1}\left(\tau^{\prime}\right), \quad \beta_{-}=\beta_{+}-z_{o} / c, \quad M_{1}=\xi_{-}^{-1}\left\{\eta^{2}\left(\xi_{-} \alpha_{+}-\xi_{+} \alpha_{-}\right)-\frac{\partial}{c d \tau^{\prime}}(\eta \tilde{\alpha})\right\} \\
\left.B(\tau)=\sqrt{\left(l_{o} / c\right)^{2}+(2 / c) \int_{0}^{\tau} d \tau^{\prime} M_{2}\left(\tau^{\prime}\right)}, \quad M_{2}=\xi_{-}^{-2} \eta\left\{\xi_{-}-\eta \tilde{\alpha}\left(\xi_{-} \alpha_{+}-\xi_{+} \alpha_{-}\right)+\eta \frac{\partial}{2 c d \tau^{\prime}} \tilde{\alpha}^{2}\right)\right\}
\end{gathered}
$$

and $z_{o}=\left(\xi_{-} \xi_{+}\right)^{-1}\left(\xi_{+}+\xi_{-}\right)$is a spatial shift of $A_{-}$with respect to the envelope $A_{+}\left(z_{o}<<l_{o}\right)$.

\subsection{Numerical simulations and discussions}

Here we numerically demonstrate quantum manipulation of a traveling light pulse for the two-color stationary light by solving Eqs. (2) and (3). For simplicity we ignore the weak decay rate $\gamma_{2}$ between the two ground states $|1\rangle$ and $|2\rangle$, and assume small level splitting $\omega_{21}$ to ignore the phase mismatch between the fields $\Psi_{+}$and $\Psi_{-}$. When the backward control field is turned off $\left(\Omega_{-}=0\right)$, Eqs. (2) and (3) satisfy slow-light wave equations (Hau et al., 1999; Turukhin et al., 2002). We note that Eqs. (2) and (3) coincide with the standing single-frequency light based on a standing wave grating in a three-level system if $g_{+}=g_{-}$with Doppler broadening (Bajcsy et al., 2003). It should be noted that Eqs. (2) and (3) show general coupled equations of standing lights whether the optical dense medium is Doppler broadened or not. At $t=t_{1}$, both control fields $\Omega_{+}$and $\Omega_{-}$are turned on. The coupled quantum fields $A_{\sigma}$ are therefore generated and they propagate and evolve together in the medium. If the stationary light condition $g_{+} / g_{-}=\Omega_{+} / \Omega_{-}=1$ is satisfied, the coupled fields $A_{\sigma}$ should be standing still with nearly the same amplitude until one control field is turned off. This condition corresponds to the appearance of the so-called dark state in the double- $\Lambda$ system (Korsunsky \& Kosachiov, 1999), which can also dramatically change the interaction of the copropagating light fields.

Compared with the standing-wave grating case (Bajcsy et al., 2003; Andre et al., 2005), the group velocity $v=c M_{1}\left(\Omega_{\Sigma}^{2} / N_{+}^{2}\right)$ in Eq. (11) can be changed by adjusting the two control fields. However, the lowest velocity is realized only if $\left|\frac{\partial}{c d \tau^{\prime}} \eta \tilde{\alpha}\right| \rightarrow 0$ and $\left(\xi_{-} \alpha_{+}-\xi_{+} \alpha_{-}\right) \rightarrow 0$ occur with complete light stoppage $(v=0)$. The fields $\mathrm{A}_{+}$and A- are strongly coupled and move or stay together with the following amplitude ratio: $\left(g_{-} / \Omega_{-}\right) A_{-}(t, z) \cong\left(g_{+} / \Omega_{+}\right) A_{+}(t, z)$. This means the field A. can be generated by the frequency shift $\omega_{-}=\omega_{41}+\delta \omega_{+}$if the frequency of the field $\mathrm{A}_{+}$is tuned to $\omega_{+}=\omega_{31}+\delta \omega_{+}$. The standing field $\hat{E}(t, z) \sim g_{+}^{-1} \Omega_{+} \Psi_{+} e^{-i \omega_{+}(t-z / c)}+g_{-}^{-1} \Omega_{-} \Psi_{-} e^{-i \omega_{-}(t+z / c)}$ is bound to the coherence moving grating (Moiseev \& Ham, 2006; Ham, 2006; Moiseev et al., 2006; Moiseev \& Ham, 2007; Ham \& Hahn, 2009) which is completely different from the standing-wave grating (Bajcsy et al., 2003; Andre et al., 2005).

At time $t=t_{2}$, we turn off one control laser $\Omega_{+}$or $\Omega_{-}$. Fig. 2 gives the switching sequence of two control fields for these two cases: (a) the control field $\Omega_{+}$is always on, while the $\Omega_{-}$is on only for $t_{1}<t<t_{2}$; (b) the control field $\Omega_{+}$is on for $0<t<t_{2}$, while the control field $\Omega_{-}$is on for $t_{1}<t$.

Fig. 3 shows numerical simulation of the two-color stationary light for case (a) mentioned above (also see Fig. 2(1)). For simplification, we set $\gamma_{2}=0$ and assume the same Rabi 

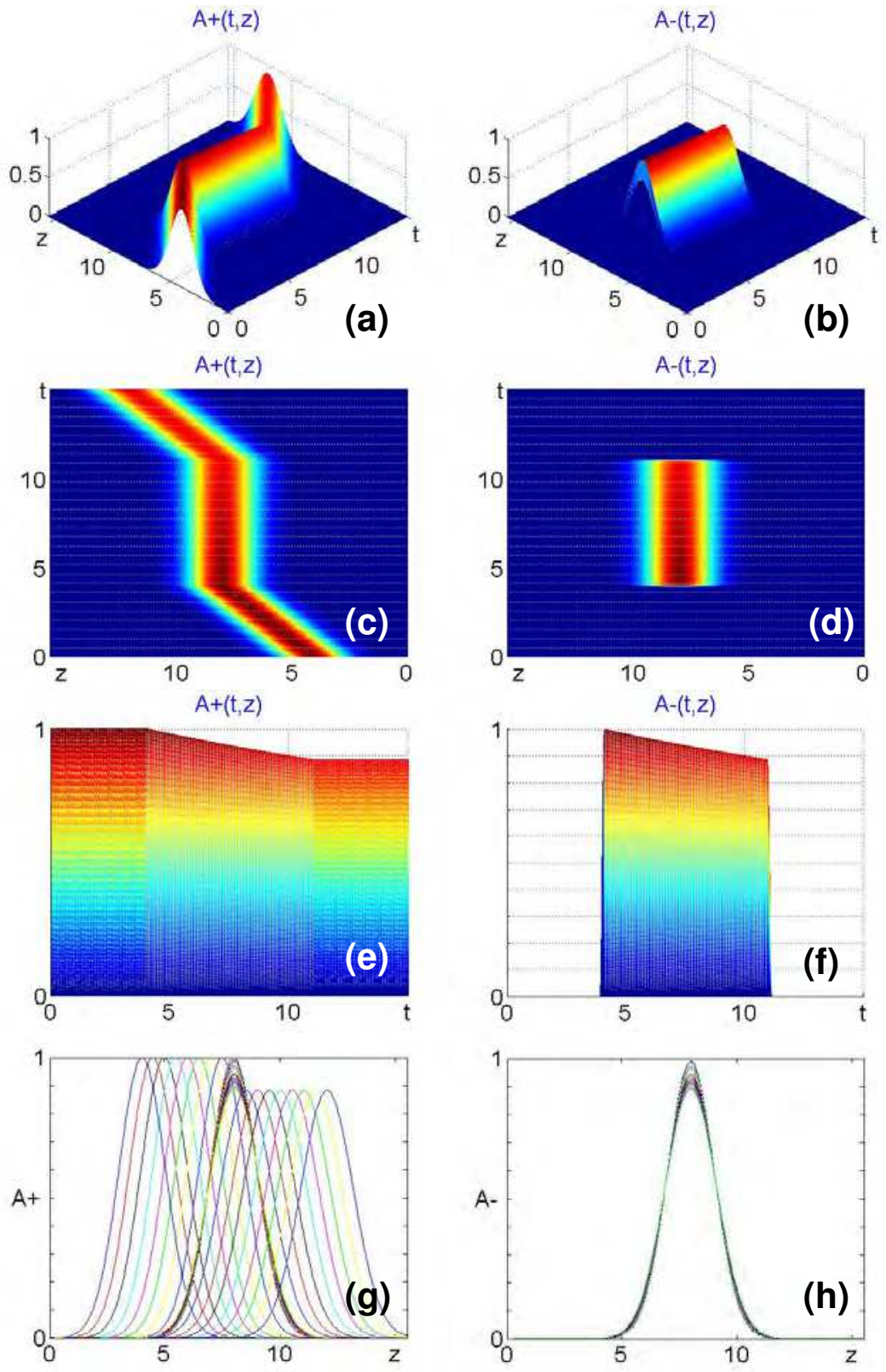

Fig. 3. Numerical simulation of two-color stationary light. The control field $\Omega_{+}$is always on, while the $\Omega_{-}$is on only for $t_{1}(4)<t<t_{2}(11)$. 

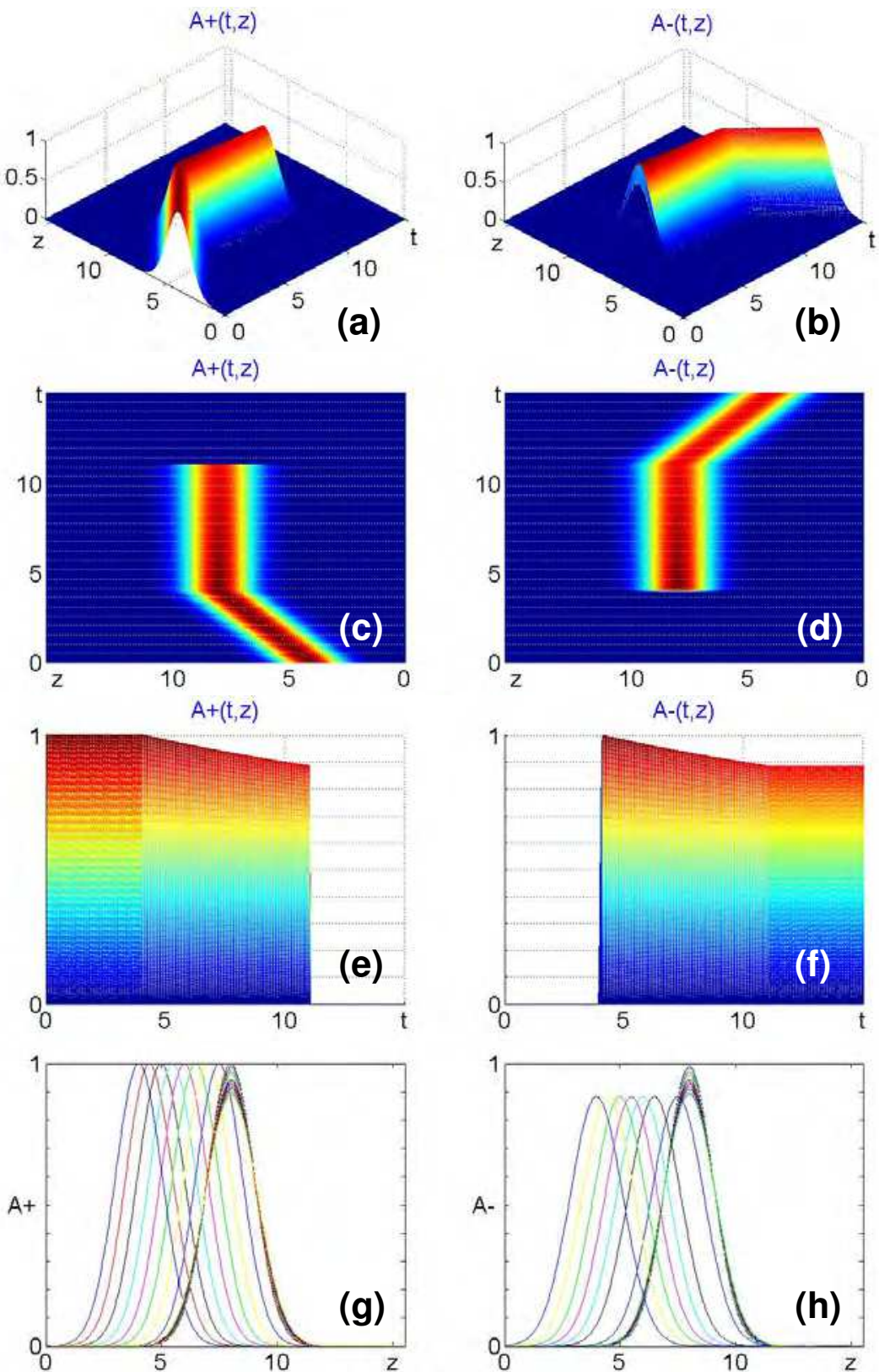

Fig. 4. Numerical simulation of two-color stationary light for wavelength conversion. The control field $\Omega_{+}$is on for $0<t<t_{2}(11)$, while the $\Omega_{-}$is on for $t_{1}(4)<t<15$. 
frequencies for the control fields. Fig. 3(c) and Fig. 3(d) show the top view of the fields $A_{+}$ and A- propagation, respectively. Fig. 3(e) and Fig. 3(f) show the temporal evolutions of the field amplitudes $A_{+}$and A., respectively. Fig. 3(g) and Fig. 3(h) show space-time evolution of the fields $A_{+}$and $A_{-}$, respectively. These figures show that when the control pulse $\Omega_{-}$is turned off at $t=t_{2}\left(t_{2}=11\right)$, the standing field A. disappears completely, and the original field $A_{+}$keeps propagation moving in the same direction at the same group velocity as it had for $0<t<t_{1}\left(t_{1}=4\right)$.

Fig. 4 shows numerical simulation of the two-color stationary light for case (b) (see Fig. 2(2)). Similar to Fig. 3, Fig. 4(c) and Fig. 4(d) show the top view of the coupled fields. Fig. 4(e) and Fig. 4(f) show the temporal evolutions of the coupled field amplitudes. Fig. 4(g) and Fig. 4(h) show space-time evolution of the coupled fields. As seen in Figs. 4(c) and 4(d), when $\Omega_{+}$is turned off at $t=t_{2}\left(t_{2}=11\right)$ while the $\Omega_{-}$is kept turned on, the quantum field $A_{+}$disappears at $t=t_{2}$, but the A. propagates in the backward direction with new carrier frequency $\omega_{-}$. Figs. 3 and 4 show that by controlling the coupling fields, the coupled fields E+ and E- can be manipulated for stationary light or frequency conversion, which has potential application for quantum nonlinear optics which needs a longer interaction time. Here we note that the maximum trapping time of stationary light is determined by the coherence decay rate $\gamma_{2}$ between the two ground states $\mid 1>$ and $\mid 2>$. Thus for practical application, we may choose an optically dense medium with a long spin decay time.

In summary, we have demonstrated two-color stationary light and quantum wavelength conversion using a quantum-mechanically reversible process between photons and atomic coherence in a double- $\Lambda$ four-level system. In this scheme, a traveling quantum field can be manipulated by simply adjusting the control fields' parameters for (1) determination of twocolor stationary light, (2) selection of propagation direction, either forward or backward, and (3) dynamic quantum frequency conversion. The present quantum manipulation of two-color stationary light can be used for enhanced nonlinear interactions between single photon fields.

\section{Quantum manipulation of MC stationary light}

If we choose a multilevel system, MC stationary light should be possible. In this section, we show quantum coherent control of multiple travelling light pulses in an optically dense medium by generalizing the approach we used in the previous section. Fig. 5 shows an energy level diagram of the present MC stationary light. The control fields with Rabi frequencies $\Omega_{m}$ copropagate forward along with the weak quantum fields $\hat{E}_{m}\left(m \in\left[1, \ldots, M_{+}\right]\right)$, whereas the other quantum fields $\widehat{E}_{n}\left(n \in\left[1, \ldots, M_{-}\right]\right)$propagate backward along with their control fields with Rabi frequencies $\Omega_{n}$. The propagation directions of fields for the effective generation and strong interaction between the fields should satisfy the phase matching condition. As shown in Fig. 5, the quantum fields $\hat{E}_{m}$ and $\hat{E}_{n}$ are nearly resonant with optical transitions $|1\rangle-|m\rangle$ and $|1\rangle-|n\rangle$, respectively. The control fields $\Omega_{m}$ and $\Omega_{n}$ have frequencies close to the frequencies of transitions $|2\rangle-|m\rangle$ and $|2\rangle-|n\rangle$, respectively (where $\Omega_{m}(t)=\Omega_{m, o}(t) e^{i \varphi_{m}}, \Omega_{n}(t)=\Omega_{n, o}(t) e^{i \varphi_{n}}$, and $\phi_{m(n)}$ are the constant phases of the fields).

We assume that initially all the atoms stay in the ground state $|1\rangle$ and only one weak probe quantum field $\widehat{E}_{l}$ enters into the medium with a forward propagation direction $k_{l}$ and the 


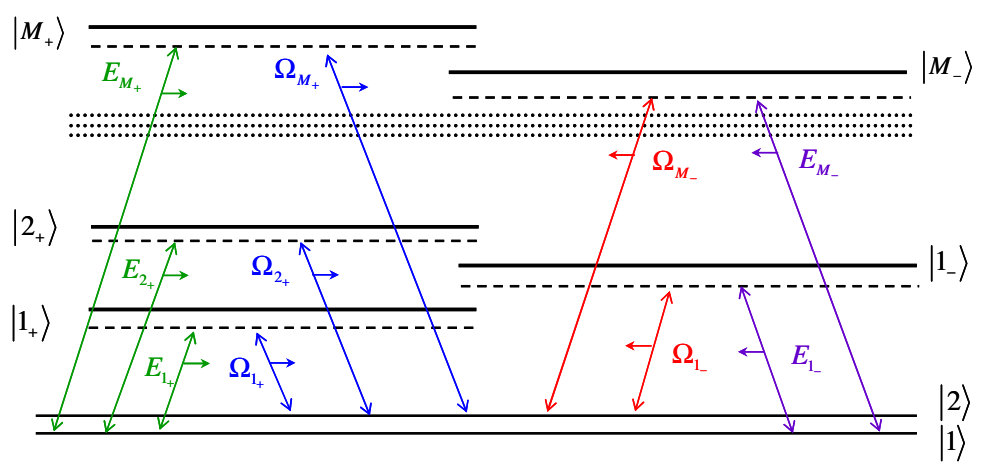

Fig. 5. Energy level diagram for MC stationary light.

atoms are driven by one classical control field $\Omega_{l} \neq 0$ with same propagation direction $K_{l}$. The initial state of light and atoms in the interaction picture is given by $|\varphi(t<<0)\rangle_{\text {in }}=\left|\varphi_{l}\right\rangle|1\rangle_{\text {atoms }}\left(|1\rangle_{\text {atoms }}=\prod_{j}\left|1_{j}\right\rangle\right.$ and $\left|\varphi_{l}\right\rangle$ are the ground state of the atoms and initial state of the probe field, respectively). The quantum field $\hat{E}_{l}$ propagates at a slow group velocity due to the control field $\Omega_{l}$, and atomic coherent wave $\left\langle\hat{P}_{12}\right\rangle$ is created between two ground states $|1\rangle$ and $|2\rangle$. After the probe pulse completely enters the medium, we switch on the additional control fields $\left(\Omega_{m}\left(t>t_{1}\right) \neq 0\right.$ and $\left.\Omega_{n}\left(t>t_{1}\right) \neq 0\right)$. Due to the ultraslow propagation of the probe field, such switching processes can be easily realized. The new atomic polarization waves with wave vectors $k_{m, n}=k_{l}-K_{l}+K_{m, n}$ (phase matching condition) which generate the new light field $\hat{E}_{m, n}$ are created. Therefore all the quantum fields become coupled with each other via atomic coherence $\left\langle\hat{P}_{12}\right\rangle$ which has the quantum information of the initial probe field $\hat{E}_{l}$. Below we will study the evolution of MC stationary light in the coherent resonance medium driven by the $\mathrm{M}_{+}+\mathrm{M}$ - control fields.

For a theoretical analysis of MC stationary light based on the multi double lambda-type scheme in Fig. 5, we introduce quantum fields $\widehat{E}_{m, n}(t, z)=\sqrt{\hbar \omega_{m, n} /\left(2 \varepsilon_{0} S\right)} \hat{A}_{m, n}(t, z) e^{-i\left(\omega_{m, n} t \mp k_{m, n} z\right)}$, where down indexes $m$ and $n$ are related to the signs $\mp$ or \pm in the equations. $\widehat{A}_{m(n)}$ are slowly varying field operators, where $\left[\widehat{A}_{p}(t, z), \widehat{A}_{q}\left(t, z^{\prime}\right)\right]=\delta_{p, q} \delta\left(z-z^{\prime}\right), \quad \hbar$ is the Planck's constant, $\varepsilon_{0}$ is the electric permittivity, and $S$ is a cross section of light beams (Loudon 2000). The interaction of the quantum fields with atoms driven by the control laser fields $\Omega_{m}(t)$ and $\Omega_{n}(t)$ is given by the following Hamiltonian in the interaction picture:

$$
\begin{aligned}
& \hat{H}(t)=\widehat{V}_{f}(t)+\widehat{V}_{b}(t)+H . C ., \\
& \widehat{V}_{f}(t)=-\hbar \sum_{j}\left\{\sum_{m=1}^{M_{+}} g_{m} \widehat{A}_{m}\left(t, z_{j}\right) e^{-i\left(\Delta_{m} t-k_{m} z_{j}\right)} \widehat{P}_{m 1}^{j}+\sum_{m=1}^{M_{+}} \Omega_{m}(t) e^{-i\left(\Delta_{m} t-\left(k_{m}-k_{0}\right) z_{j}\right)} \widehat{P}_{m 2}^{j}\right\} \\
& \widehat{V}_{b}(t)=-\hbar \sum_{j}\left\{\sum_{n=1}^{M_{-}} g_{n} \widehat{A}_{n}\left(t, z_{j}\right) e^{-i\left(\Delta_{n} t-k_{n} z_{j}\right)} \widehat{P}_{n 1}^{j}+\sum_{n=1}^{M_{-}} \Omega_{n}(t) e^{-i\left(\Delta_{n} t-\left(k_{n}-k_{0}\right) z_{j}\right)} \widehat{P}_{n 2}^{j}\right\}
\end{aligned}
$$


where $\hat{P}_{l l^{\prime}}^{j}=|l\rangle_{j j}\left\langle l^{\prime}\right|$ is an operator of the $\mathrm{j}$-th atom, $g_{l}=\mu_{l, 1} \sqrt{\omega_{l, 1} /\left(2 \varepsilon_{0} \hbar S\right)}$ is a coupling constant of photons with atoms, $\mu_{l, l}$ is a dipole moment for the transition between the states $\left|l^{\prime}\right\rangle \rightarrow|l\rangle, v_{m}=\omega_{m 1}+\Delta_{m}, v_{n}=\omega_{n 1}+\Delta_{n}$, and $k_{0}=\omega_{21} / c$.

Using Eq. (12) and adding the relaxation constants and Langevin forces associated with the atomic relaxation processes, we derive the Heisenberg equations for the atomic operators $\widehat{S}_{1 m}^{j}=\widehat{P}_{1 m}^{j} \exp \left\{i\left(\Delta_{m} t-k_{m} z_{j}\right)\right\}, \widehat{S}_{1 n}^{j}=\widehat{P}_{1 n}^{j} \exp \left\{i\left(\Delta_{n} t+k_{n} z_{j}\right)\right\}$ and $\widehat{P}_{12}^{j}$ :

$$
\begin{gathered}
\frac{\partial}{\partial t} \widehat{S}_{1 m}^{j}=-\tilde{\gamma}_{1 m} \widehat{S}_{1 m}^{j}+i g_{m} \widehat{A}_{m}\left(t, z_{j}\right)\left(\widehat{P}_{11}^{j}-\widehat{P}_{m m}^{j}\right)+i \Omega_{m} e^{-i k_{0} z_{j}} \widehat{P}_{12}^{j}+\widehat{F}_{1 m ;(1)}^{j}(t) \\
\frac{\partial}{\partial t} \widehat{S}_{1 n}^{j}=-\tilde{\gamma}_{1 n} \widehat{S}_{1 n}^{j}+i g_{n} \widehat{A}_{n}\left(t, z_{j}\right)\left(\widehat{P}_{11}^{j}-\hat{P}_{n n}^{j}\right)+i \Omega_{n} e^{i k_{0} z_{j}} \widehat{P}_{12}^{j}+\widehat{F}_{1 n ;(1)}^{j}(t) \\
\frac{\partial}{\partial t} \widehat{P}_{12}^{j}=-\gamma_{12} \hat{P}_{12}^{j}+i \sum_{m=1}^{M_{+}} \Omega_{m}^{*}(t) e^{i k_{0} z_{j}} \widehat{S}_{1 m}^{j}+i \sum_{n=1}^{M_{-}} \Omega_{n}^{*}(t) e^{-i k_{0} z_{j}} \widehat{S}_{1 n}^{j} \\
-i g_{m} \widehat{A}_{m}\left(t, z_{j}\right) \widehat{S}_{m 2}^{j}-i g_{n} \widehat{A}_{n}\left(t, z_{j}\right) \widehat{S}_{n 2}^{j}+\widehat{F}_{12 ;(0)}^{j}(t)
\end{gathered}
$$

where $\tilde{\gamma}_{m, n}=\gamma_{m, n}-i \Delta_{m, n}, \hat{F}_{1 m ;(1)}^{j}(t)=\exp \left\{i\left(\Delta_{m} t-k_{m} z_{j}\right)\right\} \widehat{F}_{1 m ;(0)}^{j}(t), \hat{F}_{1 n ;(1)}^{j}(t)=\exp \left\{i\left(\Delta_{n} t+k_{n} z_{j}\right)\right\} \widehat{F}_{1 n ;(0)}^{j}(t)$ and the fluctuations satisfy the usual properties

$$
\begin{gathered}
\left\langle\hat{F}_{p q ;(0)}^{j}(t)\right\rangle=0 \\
\left\langle\hat{F}_{1 m(n) ;(0)}^{j}(t) \hat{F}_{m(n) 2 ;(0)}^{j}\left(t^{\prime}\right)\right\rangle=\left(\gamma_{1, m(n)}+\gamma_{m(n), 2}-\gamma_{12}\right)\left\langle\hat{P}_{12}^{j}(t)\right\rangle \delta\left(t-t^{\prime}\right) \\
\left\langle\hat{F}_{1 m(n) ;(0)}^{j}(t) \hat{F}_{m(n) 1 ;(0)}^{j}\left(t^{\prime}\right)\right\rangle=2 \gamma_{1 m(n)}\left\langle\hat{P}_{11}^{j}(t)\right\rangle \delta\left(t-t^{\prime}\right) \cong 2 \gamma_{1 m(n)} \delta\left(t-t^{\prime}\right)
\end{gathered}
$$

In Eqs. (13)-(15), we ignore the influences of the weak populations of excited states $|m\rangle$ and $|n\rangle$. Thus we have the following equations for the weak field operators $\widehat{A}_{m, n}$ :

$$
\begin{gathered}
\left(\frac{\partial}{c \partial t}+\frac{\partial}{\partial z}\right) \widehat{A}_{m}(t, z)=i\left(\sqrt{n_{0} S} g_{m}^{*} / c\right) \widehat{S}_{1 m}(t, z) \\
\left(\frac{\partial}{c \partial t}-\frac{\partial}{\partial z}\right) \hat{A}_{n}(t, z)=i\left(\sqrt{n_{0} S} g_{n}^{*} / c\right) \widehat{S}_{1 n}(t, z)
\end{gathered}
$$

where $\widehat{S}_{1 m(n)}(t, z)=\left(n_{0} S\right)^{-1 / 2} \sum_{j} \widehat{S}_{1 m(n)}^{j}(t) \delta\left(z-z_{j}\right)$.

We assume a typical adiabatic condition for slow light propagation $\tilde{\gamma}_{m, n} \delta t_{0} \gg 1$ ( $\delta t_{0}$ is large enough temporal duration of the probe pulse) and ignore the fluctuation forces $\hat{F}_{m 2 ;(2)}^{j}$ and $\hat{F}_{n 2 ;(2)}^{j}$ due to the weak quantum fields. Using Eqs. (13)-(20) and introducing new field operators $\hat{\Psi}_{m, n}(t, z)=e^{ \pm i k_{0} z} \sqrt{n_{0} S} g_{m, n} \hat{A}_{m, n}(t, z) / \Omega_{m, n}(t)$, we finally obtain the following equations for $\bar{\Psi}_{m, n}$ under the slow light propagation condition $v_{m, n}(t)=c \Omega_{m, n}^{2}(t) / N g_{m, n}^{2}<<c$ : 


$$
\begin{aligned}
& \left(\frac{\partial}{\partial z}-i k_{0}\right) \widehat{\Psi}_{m}=-\xi_{m}\left\{\widehat{\Psi}_{m}-\left(1-\frac{\partial}{\mu \partial t}\right)[G(\hat{\Psi}) / \mu]\right\}-\left(\xi_{m} / \mu\right) \widehat{F}_{1 m}^{(3)} \\
& \left(\frac{\partial}{\partial z}+i k_{0}\right) \widehat{\Psi}_{n}=+\xi_{n}\left\{\widehat{\Psi}_{n}-\left(1-\frac{\partial}{\mu \partial t}\right)[G(\hat{\Psi}) / \mu]\right\}+\left(\xi_{n} / \mu\right) \widehat{F}_{1 n}^{(3)}
\end{aligned}
$$

where the fluctuation forces are:

$$
\widehat{F}_{1 n, 1 m}^{(3)}(t, z)=\left(n_{0} S\right)^{-1 / 2} \sum_{j}\left[\widehat{F}_{12,(\Sigma 2)}^{j}(t)-i \exp \left(\mp i k_{0} z_{j}\right) \mu(t) \Omega_{n, m}^{-1}(t) \widehat{F}_{1 n, 1 m ;(2)}^{j}(t)\right] \delta\left(z-z_{j}\right)
$$

Eqs. (21) and (22) can be solved by using spatial Fourier transformation. Assuming that initial field $\widehat{\Psi}_{l}(t, z)$ is determined by the probe pulse field $A_{l}(t, z)$, after some algebraic calculations we finally obtain the following solution:

$$
\widehat{\Psi}_{m,(n)}(t, z)=\widehat{\Psi}_{\operatorname{det} ; m(n)}(t, z)+\delta \hat{\Psi}_{s t ; m(n)}(t, z)
$$

where

$$
\begin{gathered}
\widehat{\Psi}_{\text {det; } m(n)}(t, z)=\int_{-\infty}^{\infty} d k \exp (i k z) T\left(t-t_{0}, k, k_{0}\right) \widehat{\psi}_{l}\left(t_{0}, k\right)=\int_{0}^{L} d z^{\prime} f_{m,(n) ; l}\left(z-z^{\prime}\right) \widehat{\Psi}_{\text {det } ;}\left(t, z^{\prime}\right) \\
\delta \widehat{\Psi}_{s t ; m,(n)}(t, z)=\int_{0}^{L} d z^{\prime} f_{m,(n) ; l}\left(z-z^{\prime}\right) \delta \widehat{\Psi}_{s t ; l}\left(t, z^{\prime}\right)+\delta \widehat{\Psi}_{s t ; m(n) ; l}(t, z) \\
f_{m(n), l}\left(z-z^{\prime}\right)=(1 / 2 \pi) \int d k \exp \left[i k\left(z-z^{\prime}\right)\right]\left[1+i \eta_{m(n), l}\left(k ; k_{0}\right)\right] \\
\eta_{n(m) ; l}\left(k ; k_{0}\right)=\left[\xi_{l}^{-1}\left(k-k_{0}\right)+\xi_{n, m}^{-1}\left(k_{0} \pm k\right)\right] /\left[1-i \xi_{n, m}^{-1}\left(k_{0} \pm k\right)\right]
\end{gathered}
$$

\section{Results and discussion}

In this section, we analyze quantum evolution of the MC field. Under the slow light condition and taking into account weak relaxation processes between the two ground levels $|1\rangle$ and $|2\rangle$, we can get the key information of quantum dynamics control from the dispersion relation:

$$
\begin{gathered}
\omega\left(t, k, k_{0}\right) \cong v(t) k-i \delta \omega_{k k}^{\prime \prime}(t) k^{2} / 2+\ldots, \\
v(t)=\omega_{k}^{\prime}=c\left[\sum_{m}^{M_{+}} \Omega_{m ; 0}^{2} / N g_{m}^{2}-\sum_{n}^{M_{-}} \Omega_{n ; 0}^{2} / N g_{n}^{2}\right]=\sum_{m}^{M_{+}} v_{m}-\sum_{n}^{M_{-}} v_{n} \\
\delta \omega_{k k}^{\prime \prime}=i \omega_{k k}^{\prime \prime}=2\left[\sum_{m, m^{\prime}}^{M_{+}} \Gamma_{m} \Gamma_{m^{\prime}}\left(\xi_{m}^{-1}-\xi_{m^{\prime}}^{-1}\right)^{2}+\sum_{n, n^{\prime}}^{M_{-}} \Gamma_{n} \Gamma_{n^{\prime}}\left(\xi_{n}^{-1}-\xi_{n^{\prime}}^{-1}\right)^{2}+\sum_{m, n}^{M_{+}, M_{-}} \Gamma_{m} \Gamma_{n}\left(\xi_{m}^{-1}+\xi_{n}^{-1}\right)^{2}\right] / \mu
\end{gathered}
$$

where $v(t)$ is the united group velocity of all the laser fields, and $-i \delta \omega_{k k}^{\prime \prime}$ is the second order dispersion term which determines the spatial broadening of the MC light pulse. 


\subsection{Traveling MC field}

If $M_{-}=0$ and $M_{+}>1$ we have the traveling MC light field with the group velocity:

$$
v(t)=v_{\text {travel }}(t)=\sum_{m}^{M_{+}} v_{m}(t)
$$

where the spatial dispersion will be

$$
\delta \omega_{k k ; \text { travel }}^{\prime \prime}=2\left\{\sum_{m, m^{\prime}}^{M_{+}} \Gamma_{m} \Gamma_{m^{\prime}}\left(\xi_{m}^{-1}-\xi_{m^{\prime}}^{-1}\right)^{2}\right\} / \mu
$$

If $\Delta_{m(m)} / g_{m\left(m^{\prime}\right)}^{2}=$ const, the spatial broadening of the traveling MC field can be minimized to

$$
\delta \omega_{k k ; \text { travel }}^{\prime \prime}=2\left\{\sum_{m, m^{\prime}}^{M_{+}} \Gamma_{m} \Gamma_{m^{\prime}}\left(1 / \xi_{m}^{0}-1 / \xi_{m^{\prime}}^{0}\right)^{2}\right\} / \mu
$$

If $\gamma_{m} / g_{m}^{2}=$ const, this value can be equal to zero.

\subsection{Stationary MC field}

Eg. (26) shows that all the laser fields propagate and evolve together with one group velocity $v(t)$ in the medium. When $v(t)=0$, that is, when all the laser fields are completely stopped, we can obtain MC stationary light:

$$
\sum_{m}^{M_{+}} \Omega_{m ; 0}^{2}(t) / g_{m}^{2}-\sum_{n}^{M_{-}} \Omega_{n ; 0}^{2}(t) / g_{n}^{2}=0
$$

This is the MC stationary light condition. Obviously, this relation generalizes the results obtained for two-color stationary light. The amplitudes of these coupled fields can be manipulated by varying the Rabi frequencies of the corresponding control fields: $\widehat{A}_{m}(t, z) / \widehat{A}_{n}(t, z) \cong \Omega_{m}(t) g_{n} /\left(\Omega_{n}(t) g_{m}\right)$. Therefore optical control of group velocity, electric field amplitudes and direction of the MC field can be easily realized by changing the control fields' amplitudes.

For a Gaussian shape of the initial input probe pulse, we have the following amplitudes $A_{m, n}$ of the MC stationary light:

$$
\left\langle A_{m(n)}(t, z)\right\rangle_{\alpha}=\frac{\Omega_{m(n)}(t) g_{l}}{\Omega_{l}\left(t_{0}\right) g_{m(n)}} \frac{l_{0}}{l_{m(n)}(t)} A_{l, 0} \exp \left\{-\gamma_{2}\left(t-t_{0}\right)-\frac{\left[z+z_{0}-v_{l} t_{0}+\delta z_{m(n)}\right]^{2}}{2 l_{m(n)}^{2}(t)}\right\} e^{i\left(\vartheta \vartheta_{l}+\varphi_{m(n)}-\varphi_{l}\right)}
$$

Eq. (32) shows that the amplitude $A_{m, n}$ can be larger than the initial probe pulse amplitude $A_{l, 0}$, if $\Omega_{m(n)}(t) / \Omega_{l}\left(t_{0}\right)>1$.

The maximum MC field stationary time is determined by relaxation constant $\gamma_{12}$ and spatial spreading of the stationary pulse shape which is determined by the dispersion term $\delta \omega_{k k}^{\prime \prime}$. Appling Eq. (31) to Eq. (29), we get the second order dispersion:

$$
\left.\delta \omega_{k k}^{\prime \prime}\right|_{v=0}=\delta \omega_{k k ; l}^{\prime \prime}(t)=2\left\{\sum_{m}^{M_{+}} v_{m}(t) \xi_{m}^{-1}+\sum_{n}^{M_{-}} v_{n}(t) \xi_{n}^{-1}\right\}
$$


Using the conditions $\operatorname{Im}\left(\xi_{m}^{-1}-\xi_{l}^{-1}\right)=0$ and $\operatorname{Im}\left(\xi_{n}^{-1}+\xi_{l}^{-1}\right)=0$, the dispersion can be minimized to zero:

$$
\sum_{m}^{M_{+}} v_{m}(t)\left(\Delta_{m} / g_{m}^{2}\right)+\sum_{n}^{M-} v_{n}(t)\left(\Delta_{n} / g_{n}^{2}\right)=0
$$

by satisfying the particular relationship to the initial probe field $E_{l}$

$$
\Delta_{m} / g_{m}^{2}-\Delta_{l^{\prime}} / g_{l}^{2}=0, \quad \Delta_{n} / g_{n}^{2}+\Delta_{l^{\prime}} / g_{l}^{2}=0
$$

From Eqs. (34) and (35), we can know that the condition for minimum spreading is independent of the total number of the control fields $M_{+}$and M.. Putting Eq. (31) into Eq. (27), we find the important relation for the minimum spatial spreading of the MC stationary light pulses:

$$
\left.\delta \omega_{k k}^{\prime \prime}\right|_{v=0} \equiv \delta \omega_{k k ; 0}^{\prime \prime}(t)=2\left\{\sum_{m}^{M_{+}} v_{m}(t) \xi_{m}^{0}+\sum_{n}^{M_{-}} v_{n}(t) \xi_{n}^{0}\right\}
$$

\subsection{MC-wavelength conversion}

After optical trapping of the initial probe pulse by using MC stationary light, it is possible to generate an arbitrary forward traveling light field $A_{m}$ from MC stationary fields by controlling the amplitudes of the forward control fields $\left(m \in\left[1, \ldots ., M_{+}\right], \Omega_{m}\left(t>t_{2}\right) \neq 0\right.$, and $\Omega_{l \neq m}\left(t>t_{2}\right)=0$ ), or to generate an arbitrary backward traveling light field $A_{n}$ by controlling the backward control fields $\left(n \in\left[1, \ldots ., M_{-}\right], \Omega_{n}\left(t>t_{2}\right) \neq 0\right.$, and $\left.\Omega_{l \neq n}\left(t>t_{2}\right)=0\right)$. Generation of some number of copropagating quantum fields $A_{m}$ or $A_{n}$ is also possible.

The electric field amplitude of the m-th component in the MC light at time $t_{\text {out }}$ on the medium output $(\mathrm{z}=\mathrm{L})$ is given by

$$
\left\langle A_{m ; o}(t)\right\rangle_{\alpha}=\sqrt{\frac{v_{m}\left(t_{\text {out }}\right)}{v_{l}\left(t_{0}\right)}} \frac{l_{0}}{l_{m}\left(t_{\text {out }}\right)} A_{l ; 0} \exp \left\{-\gamma_{2}\left(t-t_{0}\right)+i\left(\vartheta_{l}+\varphi_{m}-\varphi_{l}\right)\right\} \exp \left\{-\frac{1}{2}\left(t-t_{\text {out }}\right)^{2} /\left(\delta t_{m, \text { out }}\right)\right\}
$$

with the temporal duration $\delta t_{m, \text { travel }}=l_{m}\left(t_{\text {out }}\right) / v_{\text {travel }}\left(t_{\text {out }}\right)$. Eq. (37) shows temporal properties of the output MC light field. We note that this MC wavelength conversion may have potential applications in optical communications networks.

\section{Conclusion}

We first demonstrated the two-color stationary light and quantum wavelength conversion using quantum coherence resulting from strongly coupled slow light through EIT in a double-lambda system. Then we generalized the approach to the MC light fields in the multi double $\Lambda$ coherent atomic medium driven by the $\mathrm{M}_{+}+\mathrm{M}$ - control intensive laser fields and showed how to manipulate the MC light field within the adiabatic limit. The results show that the MC light fields can be controlled by simply adjusting the control fields' parameters for (1) MC stationary light, (2) selection of propagation direction (forward or backward), and (3) MC wavelength conversion. The maximum stationary time and minimum spatial 
spreading of the MC field have also been discussed. On-demand quantum manipulation of the MC light field can greatly increase the interaction time of the light and medium, and holds promise for applications in optical buffer, controllable switching, and quantum optical information processing.

We acknowledge that this work was supported by the CRI program (Center for Photon Information Processing) of the Korean Ministry of Education, Science and Technology via National Research Foundation.

\section{References}

Alzetta, G.; Gozzini, A.; Moi, L. \& Oriols, G. (1976). An experimental method for the observation of r.f. transitions and laser beat resonances in oriented sodium vapor. Nuovo Cimento B, Vol. 36, (1976) 5-20

Andre, A; Bajcsy, M; Zibrov, A. X. \& Lukin, M. D. (2005). Nonlinear optics with stationary pulses of light. Phys. Rev. Lett., Vol. 94, No. 6, (2005) 063902, ISSN 0031-9007

Arimondo, E \& Orriols, G. (1976). Nonabsorbing atomic coherences by coherent two-photon transitions in a three-level optical pumping. Nuovo Cimento Letters, Vol. 17, No. 6, (1976) 333-338

Bajcsy, M.; Zibrov, A. S. \& Lukin, M. D. (2003). Stationary pulses of light in an atomic medium. Nature (London), Vol. 426, (2003) 638-641, ISSN 0028-0836

Boller, K. -J; Imamolu, A. \& Harris, S. E. (1991). Observation of electromagnetically induced transparency. Phys. Rev. Lett., Vol. 66, No. 20, (1991) 2593-2596, ISSN 0031-9007

Fleischhauer, M. \& Lukin, M. D. (2000). Dark-state polaritons in electromagnetically induced transparency. Phys. Rev. Lett., Vol. 84, No. 22, (2000) 5094-5097, ISSN 0031-9007

Fleischhauer, M.; Imamoglu, A. \& Marangos, J. P. (2005). Electromagnetically induced transparency: optics in coherent media. Rev. Mod. Phys., Vol. 77, No. 2, (2005) 633673, ISSN 0034-6861

Gray, H. R.; Whitley, R. M. \& Stroud, Carlos R., Jr. (1978). Coherent trapping of atomic populations. Opt. Lett., Vol. 3, (1978) 218-220, ISSN 0146-9592

Ham, B. S.; Hemmer, P. R. \& Shahriar, M. S. (1997). Efficient electromagnetically induced transparency in a rare-earth doped crystal. Opt. Commun., Vol. 144, (1997) 227-230, ISSN 0030-4018

Ham, B. S.; Shahriar, M. S.; Kim, M. K. \& Hemmer, P. R. (1997). Frequency-selective timedomain optical data storage by electromagnetically induced transparency in a rareearth-doped solid. Opt. Lett., Vol. 22, No. 24, (1997) 1849-1851, ISSN 0146-9592

Ham, B. S.; Shahriar, M. S.; Kim, M. K. \& Hemmer, P. R. (1998). Spin coherence excitation and rephasing with optically shelved atoms. Phys. Rev. B, Vol. 58, No. 18, (1998) R11825-R11828, ISSN 1098-0121

Ham, B. S. \& Hemmer, P. R. (2000). Coherence switching in a four-level system: quantum switching. Phys. Rev. Lett., Vol. 84, No. 18, (2000) 4080-4083, ISSN 0031-9007

Ham, B. S. (2006). Spatiotemporal quantum manipulation of travelling light: Quantum transport. Applied Physics Letters, Vol. 88, No. 12, (2006) 121117, ISSN 0003-6951

Ham, B. S. \& Hahn, J. (2009). Ultralong trapping of light using double spin coherence gratings. arXiv: 0901.1082v2, (2009)

Harris, S. E. (1989). Lasers without inversion: Interference of lifetime-broadened resonances. Phys. Rev. Lett., Vol. 62, No. 9, (1989) 1033-1036, ISSN 0031-9007 
Harris, S. E.; Fieldm, J. E. \& Imamoglu, A. (1990). Nonlinear optical processes using electromagnetically induced transparency. Phys. Rev. Lett., Vol. 64, No. 10, (1990) 1107-1110, ISSN 0031-9007

Harris, S. E. (1997). Electromagnetically induced transparency. Phys. Today, Vol. 50, No. 7, (1997) 36-42; ISSN 0031-9228

Hau, L. V.; Harris, S. E.; Dutton, Z. \& Behroozi, C. H. (1999). Light speed reduction to 17 metres per second in an ultracold atomic gas. Nature, Vol. 397, (1999) 594-598, ISSN 0028-0836

Kasapi, A.; Jain, M.; Yin, G. Y. \& Harris, S. E. (1995). Electromagnetically induced transparency: propagation dynamics. Phys. Rev. Lett., Vol. 74, No. 13, (1995) 24472450, ISSN 0031-9007

Kocharovskaya, O., Rostovtsev, Y. \& Scully, M. O. (2001). Stopping light via hot atoms. Phys. Rev. Lett., Vol. 86, No. 4, (2001) 628-631, ISSN 0031-9007

Korsunsky, E. A. \& Kosachiov, D. V. (1999). Phase-dependent nonlinear optics with doublelambda atoms. Phys. Rev. A, Vol. 60, No. 6, (1999) 4996-5009, ISSN 1050-2947

Liu, C.; Dutton, Z.; Behroozi, C. H.; Hau, L. V. \& Harris, S. E. (2001). Observation of coherent optical information storage in an atomic medium using halted light pulses. Nature, Vol. 409, (2001) 490-493, ISSN 0028-0836

Loudon, R. (2000). The Quantum Theory of Light. Oxford University Press, Oxford, ISBN 0198501765

Lukin, M. D. \& Imamoglu, A. (2000). Nonlinear optics and quantum entanglement of ultraslow single photons. Phys. Rev. Lett., Vol. 84, No. 7, (2000) 1419-1422, ISSN 0031-9007

Lukin, M. D. \& Imamoglu, A. (2001). Controlling photons using electromagnetically induced transparency. Nature, Vol. 413, (2001) 273-276, ISSN 0028-0836

Marangos, J. P. (1998). Topical review electromagnetically induced transparency. J. Modern Opt., Vol. 45, No. 3, (1998) 471-503, ISSN 0950-0340

Moiseev, S. A. \& Ham, B. S. (2006). Quantum manipulation of two-color stationary light: Quantum wavelength conversion. Phys. Rev. A, Vol. 73, No. 3, (2006) 033812, ISSN 1050-2947

Moiseev, S. A. \& Ham, B. S. (2006). Quantum control and manipulations of three-color stationary light. J. Korean Phys. Soc., Vol. 48, No. 4, (2006) 540-545, ISSN 0374-4884

Moiseev, S. A.; Chen, Y. \& Ham, B. S. (2006). Numerical analysis of stationary light for potential applications of a quantum interface. J. Korean Phys. Soc., Vol. 49, No. 6, (2006) 2293-2302, ISSN 0374-4884

Moiseev, S. A. \& Ham, B. S. (2007). Quantum control and manipulation of multi-color light fields. Optics and Spectroscopy, Vol. 103, No. 2, (2007) 210-218, ISSN 0030-400X

Nielsen, M. A. \& Chuang, I. L. (2000). Quantum Computation and Quantum Information. Cambridge University Press, Cambridge, ISBN 0521635039

Padmabandu, G. G.; Welch, G. R.; Shubin, I. N.; Fry, E. S.; Nikonov, D. E.; Lukin, M. D. \& Scully, M. O. (1996). Laser oscillation without population inversion in a sodium atomic beam. Phys. Rev. Lett., Vol. 76, No. 12, (1996) 2053-2056, ISSN 0031-9007

Phillips, M. C.; Wang, H. L.; Rumyantsev, I.; Kwong, N. H.; Takayama, R. \& Binder, R. (2003). Electromagnetically induced transparency in semiconductors via biexcition coherence. Phys. Rev. Lett., Vol. 91, No. 18, (2003) 183602, ISSN 0031-9007 
Schmidt, H. \& Imamoglu, A. (1996). Giant Kerr nonlinearities obtained by electromagnetically induced transparency. Opt. Lett., Vol. 21, (1996) 1936-1938, ISSN 0146-9592

Scully, M. O.; Zhu, S. Y. \& Gravrielides, A. (1989). Degenerate quantum-beat laser: Lasing without inversion and inversion without lasing. Phys. Rev. Lett., Vol. 62, No.24, (1989) 2813-2816, ISSN 0031-9007

Scully, M. O. (1991). Enhancement of the index of refraction via quantum coherence. Phys. Rev. Lett., Vol. 67, No. 14, (1991) 1855-1858, ISSN 0031-9007

Scully, M. O. \& Zhu, S. Y. (1992). Ultra-large index of refraction via quantum interference. Opt. Commun., Vol. 87, (1992) 134-138, ISSN 0030-4018

Scully, M. O. \& Zubairy, M. S. (1997). Quantum Optics. Cambridge University Press, Cambridge, England, ISBN 0521435951

Turukhin, A. V.; Sudarshanam, V. S; Shahriar, M. S.; Musser, J. A.; Ham, B. S. \& Hemmer, P. R. (2002). Observation of ultraslow and stored light pulses in a solid. Phys. Rev. Lett., Vol. 88, No. 2, (2002) 023602, ISSN 0031-9007

Zibrov, A. S.; Matsko, A. B.; Kocharovskaya, O; Rostovtsev, Y. V; Welch, G. R. \& Scully, M. O. (2002). Transporting and time reversing light via atomic coherence. Phys. Rev. Lett., Vol. 88, No. 10, (2002) 103601, ISSN 0031-9007 


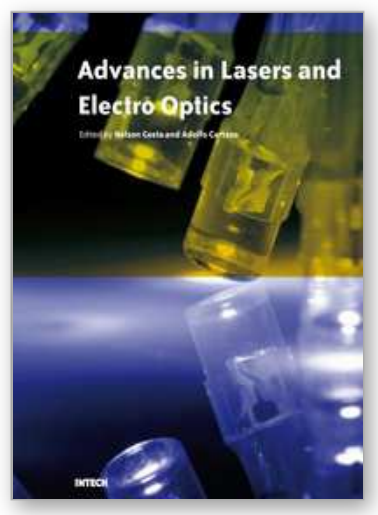

\author{
Advances in Lasers and Electro Optics \\ Edited by Nelson Costa and Adolfo Cartaxo
}

ISBN 978-953-307-088-9

Hard cover, 838 pages

Publisher InTech

Published online 01, April, 2010

Published in print edition April, 2010

Lasers and electro-optics is a field of research leading to constant breakthroughs. Indeed, tremendous advances have occurred in optical components and systems since the invention of laser in the late $50 \mathrm{~s}$, with applications in almost every imaginable field of science including control, astronomy, medicine, communications, measurements, etc. If we focus on lasers, for example, we find applications in quite different areas. We find lasers, for instance, in industry, emitting power level of several tens of kilowatts for welding and cutting; in medical applications, emitting power levels from few milliwatt to tens of Watt for various types of surgeries; and in optical fibre telecommunication systems, emitting power levels of the order of one milliwatt. This book is divided in four sections. The book presents several physical effects and properties of materials used in lasers and electro-optics in the first chapter and, in the three remaining chapters, applications of lasers and electro-optics in three different areas are presented.

\title{
How to reference
}

In order to correctly reference this scholarly work, feel free to copy and paste the following:

Yi Chen, Serguei Andreevich Moiseev and Byoung Seung Ham (2010). Multicolor Stationary Light, Advances in Lasers and Electro Optics, Nelson Costa and Adolfo Cartaxo (Ed.), ISBN: 978-953-307-088-9, InTech, Available from: http://www.intechopen.com/books/advances-in-lasers-and-electro-optics/multicolor-stationarylight

\section{INTECH}

open science | open minds

\section{InTech Europe}

University Campus STeP Ri

Slavka Krautzeka 83/A

51000 Rijeka, Croatia

Phone: +385 (51) 770447

Fax: +385 (51) 686166

www.intechopen.com

\section{InTech China}

Unit 405, Office Block, Hotel Equatorial Shanghai

No.65, Yan An Road (West), Shanghai, 200040, China

中国上海市延安西路65号上海国际贵都大饭店办公楼 405 单元

Phone: +86-21-62489820

Fax: $+86-21-62489821$ 
(C) 2010 The Author(s). Licensee IntechOpen. This chapter is distributed under the terms of the Creative Commons Attribution-NonCommercialShareAlike-3.0 License, which permits use, distribution and reproduction for non-commercial purposes, provided the original is properly cited and derivative works building on this content are distributed under the same license. 